\title{
The Study of Smartphone APP Interface Design \\ DongRui
}

School of Arts, Xi' an University, Xi' an710000, China.

397369046@qq.com

Keywords:Smartphone; APP; Interface; Limitation; Basis

\begin{abstract}
As the popularity of the smartphone, APP for smartphone has been an important industry of Mobile Internet. In this condition, users of this phone concerned much more about the experience of APP's interface. In this paper, the author based on lots of survey, summarize a serial of useful theoretical basis for the developing of the APP interface.
\end{abstract}

\section{Introduction}

According to the data from the Report of China Internet Development Statistics, $36^{\text {th }}$, up to June $30^{\text {th }}, 2015$,the total of the Mobile Internet users in China had reached to 594,000,000, which compared to the number up to December $31^{\text {th }}, 2014$,had an apparent increasement of 36,790,000(Fig.1).

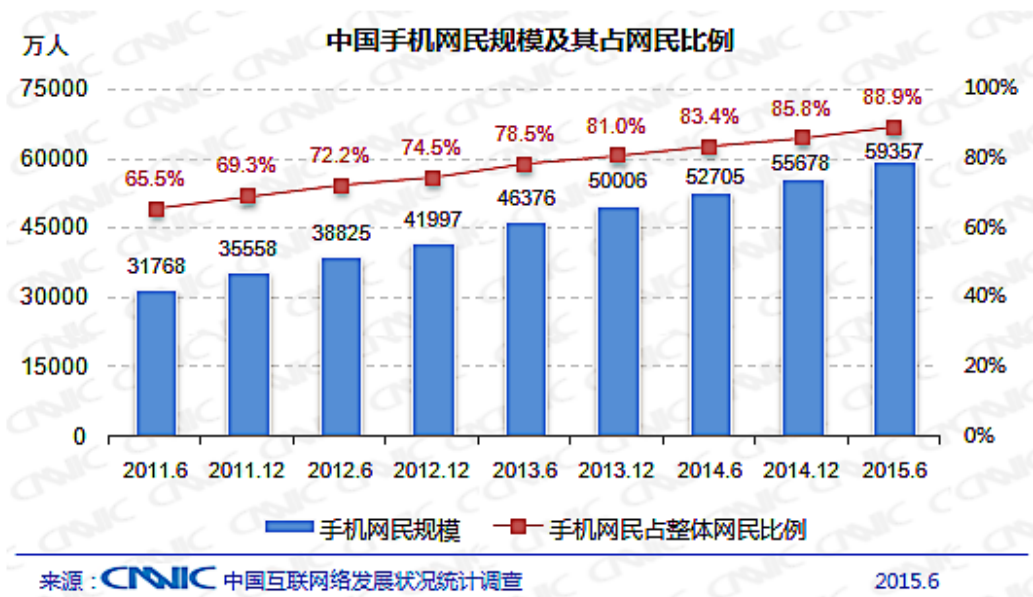

Fig.1 Chinese mobile phone user's scale and proportion

Because of the rapid spread of the $3 / 4 \mathrm{G}$ network for smartphone, the dependence of people's everyday life for the smartphone will be strenthen. On the basis of Chinese IT Research Report(Fig.2),both of the installation and using of the smartphone APP has a apparent increasement in China.

Summarice speaking, the smartphnoe and the APP has already been an important part of the people's everyday life[2]. But, with the rapid popularity of the APP, people's requirement to the APP is not the same as it yesterday,their requirement to the APP interface is increasing at the same time with a very high speed.
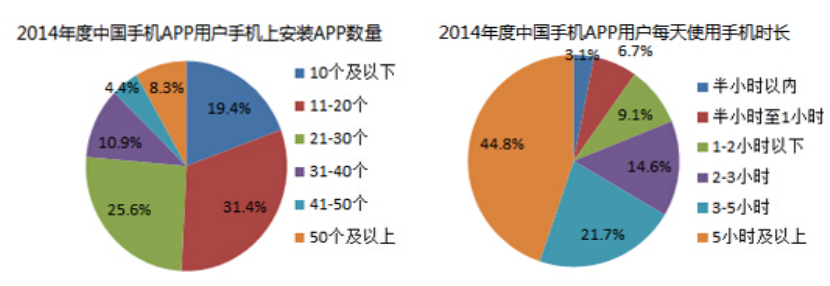

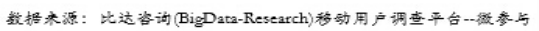

Fig.2 The number of users install the phone APP and the use of the mobile phone everyday 


\section{Definition of Smartphone and Smartphone APP Interface}

\section{A.Definition of Smartphone}

Smartphone are this phone, have independent operating system, apart from can make a telephone call, send a text message, they can also download and install the customized software.

\section{B.Definition of Smartphone APP interface}

Because the mobile phones are different from each other, so the design style of the APP interface are also in many types. As far as the smartphone APP interface concerned, they are not only the graphical user interface,generally speaking, they can be divided into 3 classes,such as Logical User Interface(LUI), Physical User Interface(PUI) and Graphical User Interface.

\section{Research on the Factors Affect the Interface Designing Come from UESR Level}

Because of the light body,easy carry,simple operation, users can use the smartphone to surf the Internet, communicate with each other, shopping at everywhere (Fig.3).It makes a lot of convenience, but all of these can't be realized without the APP. These APP not only make our everyday life much more colorful,but also give us a high qulity life with extended friend circle and more and more hobby. From these, we can draw the certain conclusion that the number of the APP will not stop its increasing, and has an abundant, convenient, intresting content will be the main trend in the develop way of the APP, and all of these must be suppoted by a fantastic APP interface.

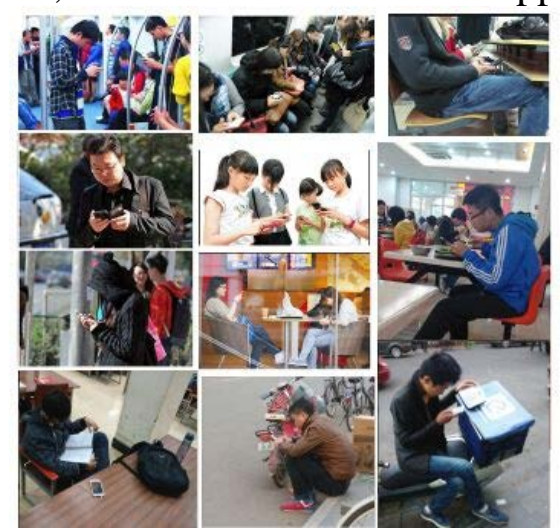

Fig.3 User use the mobile phone in different situation

\section{A. Study of the User Age Factor}

Table.1 Survey of the smartphone user's age group

\begin{tabular}{|c|c|c|}
\hline Project & Category & Proportion \\
\hline \multirow{3}{*}{ Gender } & Male & $54.4 \%$ \\
\cline { 2 - 3 } & Female & $45.6 \%$ \\
\hline \multirow{4}{*}{ Age } & $\leqslant 18$ & $5.8 \%$ \\
\cline { 2 - 3 } & $19-24$ & $43.3 \%$ \\
\cline { 2 - 3 } & $25-29$ & $25.1 \%$ \\
\cline { 2 - 3 } & $30-49$ & $24.2 \%$ \\
\cline { 2 - 3 } & $\geqslant 50$ & $1.6 \%$ \\
\hline
\end{tabular}

Data in the table.1 come from the BigData-Research consulting mobile survey data report of the China IT Research Center. From the table, we can easily find that the main user of the smartphone drop in the age group of 18-30. So when we design an APP interface, we should take this age group users'habit into consideration. 


\section{B. Study of the User's Purpose Factor}

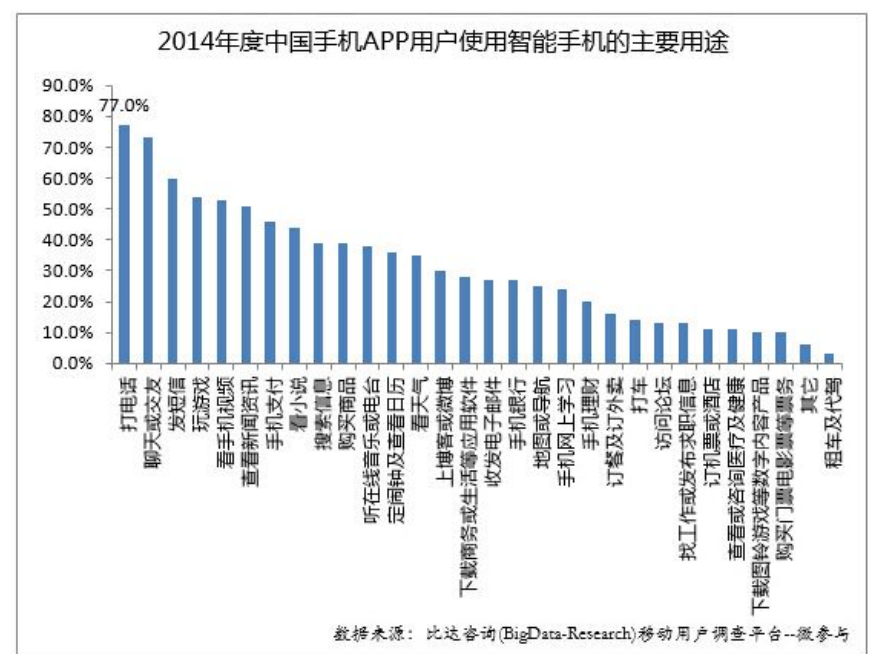

Fig.4 Survey of the user's using purpose

Like the data in the table.1, from these data we can see most of the smartphone users use the smartphone to social interaction and communication. But appart from the traditional making telephone calls and sending text message, chattingor making friends(74.6\%), playing games(55.8\%), watching the video(55.2\%), reading the news(53.2\%),etc...are all with a high frequency of everyday using.

In addition, from the data above, we can also know that,the APP users are using continuous to be diversifying and colorful. Under this condition, if the enterprise want to occupy a larger market effectively and take more and more stable customer, needless to say,they must take the customer's diversifying requirement into consideration.

\section{Research on the Factors Affect the Interface Designing Come from Phone Level}

\section{A. Study of the Technology Factor}

Technology factors is the biggest one have effect on the Interface of the interaction between the user and the smartphone.No matter the material, the hardware or the operation system, affect the APP interface design directly, so we must make good use of the current technology.

Firstly, as far as the size factor concerned, smartphone is smaller than the PC screen, so the smartphone can contain less information than the PC.But it bigger that the traditional phone and contain more information than the traditional phone. So we must notice this feature, and highlight it when designing the interface.

Secondly, in terms of the performance of screen, the smartphone can't catch up with the PC, and whatever its color of the screen, its resolution or its sharpness, affect the user's experience of the touch interaction interface directly.Needless to say, this factor also need be taken into our consideration.

Thirdly,the operation system of the smartphone is also of great significance. Currently, Android, IOS, Windows are the most popular system int the market. Usually, differnet system may result in different performance of the smartphone's input interface. So, we must think about this factor, to give the users a flexible and comfortable using experience as good as we can.

Last but not least, with the developing of the smartphone sensing technology, we can get a lot of interactive mode. For example, some APP designer combined the gravitational acceleration sensor with the game perfectly, it had changed the traditional game's operation. So when we designing the interface, we'd better know as much about the function of the sensors the smartphone have equipped as we can, only by this can we seek a novel interaction for the users. 


\section{B. Study of the Situation Factor}

To the users, different situation may have different influence on their using of the phone. So give the users a good interaction between the user and the smartphone, it is of great significance to take this factor into consideration when we designing the interface of the APP, and perfect it as good as we can.

\section{Study of the Interface Design Style Factor}

1)Skeuomorph

Simplified to say, skeuomorph is that, according to the feature of the product itself, combined with a lot of visual factors, like bright, material, texture and so on, to create a vivd appearance. Appart from this, no matter the visual interaction or the operation are all like the real items in the world. It can make the operation of the interface much easier.

2)Flat Design

Flat Design is a newly designing style with the idea that no use of any modify,shadows, gradients and so on in the whole design procedure. It with the advantage that easy using, more focusing and simplifying.

\section{Summary}

Overall, APP interface designing is not a simple thing, regardless of the user level or the smartphone level, we should take all possibile factors into consideration when we design it. Maybe just a small factor can affect the whole performance of the Interface.

\section{References}

[1]Niels henze,Sysanne boll. Release your app on Sunday eve: finding the best time to deploy apps[C] Mobile Life Centre. Proceedings of 13th International Conference on Mobile HCI. New York:ACM,2011:581-585.

[2] Susanne Ramadan Shunnaq. TheInvisibleThreat:Smart-phoneOveruse Among

Undergraduate EnglishLanguageand Literature Majors atSultan Qaboos University [A]. Global Research \& Development Service. Conference Proceedings of

the 4th International Conference on Teaching, Education and Learning (ICTEL)[C].Global Research \& Development Service:,2015:2.

[3]Ka C.Chan,Jayantha Katupitiya,Joshua W.Hanrahan,Corey J.Jackson,Dylan Rose. An Emergency Communication System for an Agricultural Autonomous Vehicle[A].

CBEES.Proceedings of 2014 International Conference on Intelligent Agriculture[C].CBEES:,2014:7.

[4] Baydaa Fadhil Dhahir. Android Application Simulation for Prototyping Wireless NanoNetworks Communication[D].Hunan University,2011.

[5]Ramesh Shrestha. Research and Implementation of Android-based Location Sharing System[D].Harbin Engineering University,2012. 